\title{
Control of a Single Phase Inverter with Multiple Modulation Strategies Based on Plant Inversion
}

\author{
R.Ramos, D Serrano, J.A.Oliver, J.A.Cobos
}

\section{INTRODUCTION}

Most of the switched converters currently used are based on the application of a switching pattern that is periodically repeated. As it is well known, the time varying nature of this switched system is removed by applying averaging over one switching cycle. In this way, the problem is reduced to the control of a non-linear system that can be further linearized around an operating point to apply well known concepts from linear control theory.

The case of the switched converter under consideration, schematically presented in Fig.1, is based on the flying capacitor converter [1], with the distinctive feature that in this case the average voltage on the flying capacitor is regulated at $0.8 V_{g}$, where $V_{g}$ is the input voltage, instead of being set at $V_{g} / 2$. The flying capacitor is also used as the energy buffer component to handle the double-line-frequency power ripple required for low input current ripple at line frequency. In this topology, the charge balance in storage capacitor $\left(C_{s}\right)$ is not done at switching frequency but at line frequency, this allows to control the input and output current. The six switches allow connecting the source and the storage capacitor to the LC filter in different ways so that the internal power processing is minimized [2], [3]. These set of switches can be commuted in different patterns (sequences) [4] that will be able to synthesize the desired inductor current, $i_{L}$, and the desired input current, $i_{g}$. The storage capacitor current will be given by applying energy balance during the switching cycle.

The application of different switching sequences (operation modes) along the line cycle makes the control more complicated; due to the converter has different plant for each

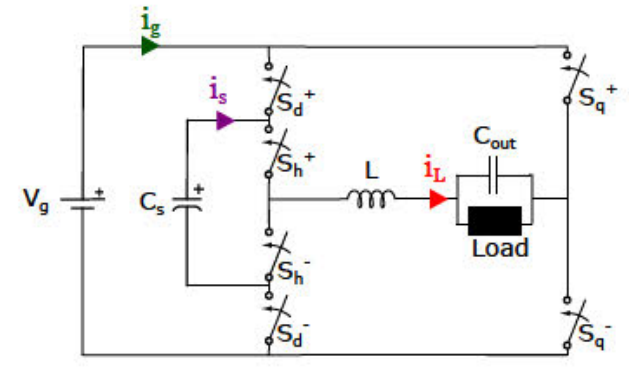

Figure 1. Simplified schematic of the flying capacitor inverter

sequence. Applying averaging over a switching cycle removes the fast-scale time varying nature of the converter [5], but since different switching sequences are applied depending on the operating conditions [4], the system can be considered a hybrid system.

\section{PRoblem Statement}

A "switching pattern" (or "operation mode") is defined as a sequence of switching actions in a given order over a switching cycle. In the switching cycle, there will be two independent times, $\left(t_{1}, t_{2}\right)$ that will determine unambiguously the switching pattern, as shown in one of the many operation modes that can be proposed, in Fig. 2. The converter operates in Boundary Conduction Mode (BCM), and because of that, the averaged current through the inductor and the input current for every specific pattern $\mathrm{k}$, will depend on the two independent times, $t_{1}$ and $t_{2}$, algebraically according to:

$$
\begin{aligned}
& <i_{L}>=f_{k}\left(t_{1}, t_{2}, x, V_{g}\right) \\
& <i_{g}>=g_{k}\left(t_{1}, t_{2}, x, V_{g}\right)
\end{aligned}
$$

Where $f_{k}$ and $g_{k}$ are non-linear algebraic functions of $t_{1}$ and $t_{2}$, the state, $x=\left(v_{s}, v_{o}\right)$, and the input voltage $V_{g}$, and $k=$ $\ldots,-2,-1,0,1,2, \ldots$ is the sequence selected for each specific angle of the line cycle. Fig. 2 shows one of these sequences, in 


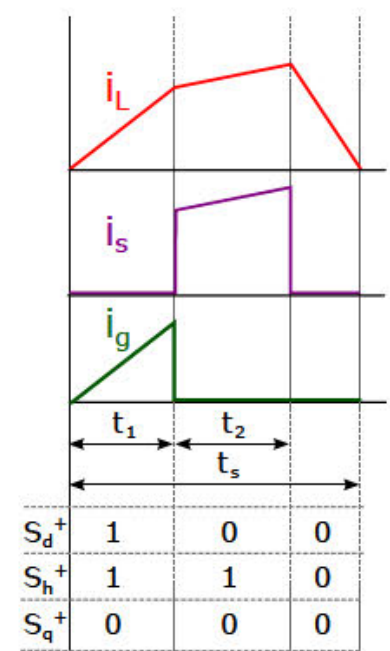

Figure 2. Trapezoidal Tri-buck mode (IV)

this particular case the inductor current and the input current can be defined as:

$$
\begin{aligned}
<i_{L}> & =\frac{\frac{1}{2} m_{1}\left(1+\frac{m_{1}}{m_{3}}\right) t_{1}^{2}+\frac{1}{2} m_{2}\left(1+\frac{m_{2}}{m_{3}}\right) t_{2}^{2}}{t_{1}\left(1+\frac{m_{1}}{m_{3}}\right)+t_{2}\left(1+\frac{m_{2}}{m_{3}}\right)}+ \\
& +\frac{m_{1}\left(1+\frac{m_{2}}{m_{3}}\right) t_{1} t_{2}}{t_{1}\left(1+\frac{m_{1}}{m_{3}}\right)+t_{2}\left(1+\frac{m_{2}}{m_{3}}\right)} \\
& <i_{g}>=\frac{\frac{1}{2} m_{1}\left(1+\frac{m_{1}}{m_{3}}\right) t_{1}^{2}}{t_{1}\left(1+\frac{m_{1}}{m_{3}}\right)+t_{2}\left(1+\frac{m_{2}}{m_{3}}\right)}
\end{aligned}
$$

Where the slopes $m_{1}, m_{2}$ and $m_{3}$ are the voltage applied across the inductor divided by the inductor value. The voltage applied across the inductor is determined by the switching pattern:

$$
v_{L}=V_{g} S_{d} \bar{S}_{q}+v_{s} \bar{S}_{d} S_{h}-V_{g} \bar{S}_{d} S_{q}-v_{s} S_{d} \bar{S}_{h}-v_{o}
$$

where $S_{i}$ is equal to 1 if $S_{i}^{+}$is ON and 0 if it is OFF.

In principle, for a given reference input current and reference output current, it will be possible to determine if for the sequence $k$ there exists a solution, and, in that case, to determine $t_{1}$ and $t_{2}$ that will provide the desired values for the input and output current. $t_{1}$ and $t_{2}$ are calculated solving these two non-linear equations for each sequence:

$$
\begin{gathered}
i_{L}^{r e f}=f_{k}\left(t_{1}, t_{2}, x, V_{g}\right) \\
i_{g}^{r e f}=g_{k}\left(t_{1}, t_{2}, x, V_{g}\right) \\
i_{1}=i_{g}^{r e f} \\
i_{3}=i_{L}^{r e f}-i_{g}^{r e f}-i_{s} \\
t_{1}=2 \sqrt{\frac{i_{1} i_{3}}{m_{1} m_{3}}}\left[\left(1-\frac{m_{1}}{m_{2}}\right) \sqrt{\frac{i_{1} m_{3}}{i_{3} m_{1}}}+1+\frac{m_{3}}{m_{2}}\right]
\end{gathered}
$$

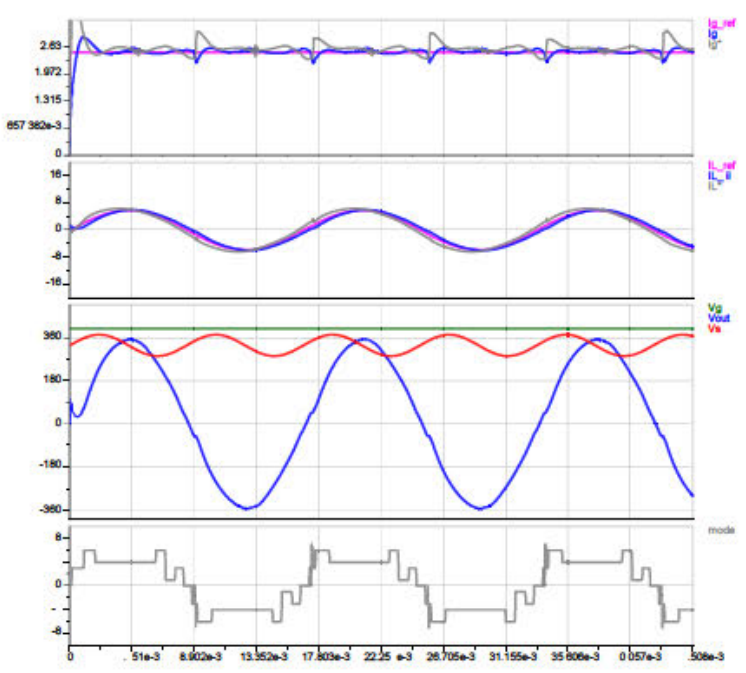

Figure 3. From top to bottom:Input current,Output current,Voltages and mode applied

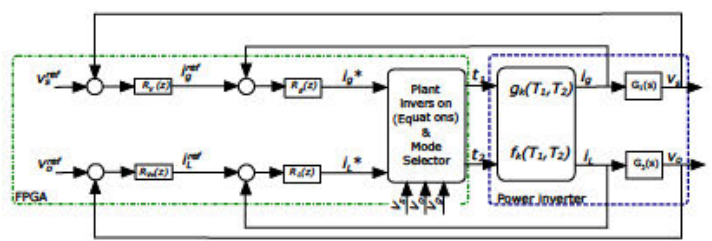

Figure 4. Complete control scheme

$$
t_{2}=t_{1} \frac{m_{3} \sqrt{\frac{i_{3} m_{1}}{i_{1} m_{3}}}-m_{1}}{m_{2}}
$$

Figure 3 shows the proper functioning of the plant inversion block, $i_{g}^{*}$ and $i_{L}^{*}$ are the references for the plant inversion. To correct small deviation from the desired values due to errors in the identification of the plant (tolerances of components, measurement errors, etc.) feedback loops were included.

The complete control scheme is shown in Fig. 4, where there is a block, called "Equations", that is in charge of selecting the right sequence and calculating the times $\left(t_{1}, t_{2}\right)$ that solves (6) and (7) and synthesizes the desired output and input current, as shown in equations (10) and (11) for the particular case of the "tri-buck" mode.

Considering the differential equations of the output and for each sequence that synthesizes the desired reference output current and the desired input current, the control problem will be reduced to:

$$
\begin{gathered}
v_{s} C_{s} \frac{d v_{s}}{d t}=v_{g} i_{g}^{r e f}-v_{o} i_{o} \\
C_{o} \frac{d v_{o}}{d t}=i_{L}^{r e f}-i_{o}
\end{gathered}
$$




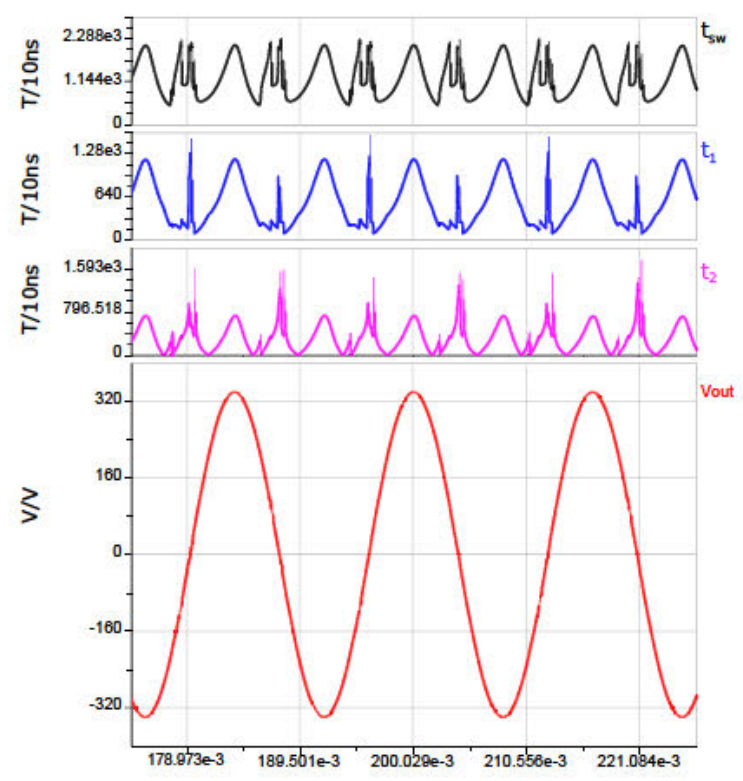

Figure 5. From top to bottom:Switching period, $t_{1}, t_{2}$, output voltage

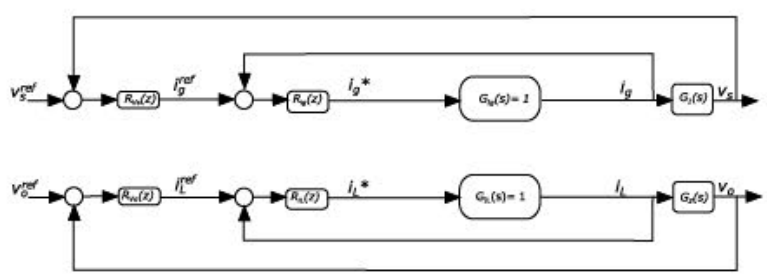

Figure 6. Simplified control scheme

It can be noticed that the output voltage can be controlled using $i_{g}^{r e f}(12)$ and the storage capacitor voltage can be controlled using $i_{L}^{r e f}(13)$, and both controllers are independent, because of the plant inversion. This method has been used to control power converters [6] and [7]. This is shown in Fig.6, where the upper control diagram represents the control of the storage capacitor voltage and the lower control diagram represents the output voltage control loop. Additionally, the design of the controllers is independent on the switching sequence; due to that the same controllers can be used along the whole line cycle.

\section{CONTROL LOOP DESIGNS}

As shown in Fig.6, the control problem is simplified to two independent first order SISO (single input single output) systems. The criterion for the design of each control loop is described below.

\section{A. Current Loops}

Due to the plant inversion, the behavior of the two current control loops will be equivalent. The objective of these control loops is to regulate the average value of each current with a fast dynamic control loop. As the converter operates in BCM

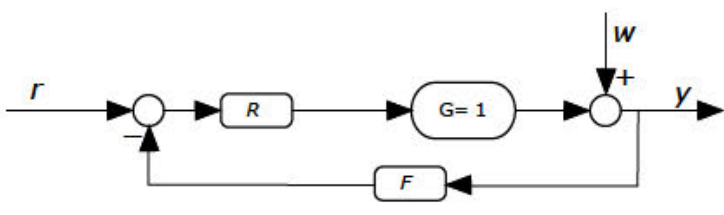

Figure 7. Simplified current control scheme

the inductor current has a large ripple, that should be neglected by the control. It is not straightforward to determine the instant in which the value of the instantaneous current is similar to its average value in a switching cycle, and it is not possible to take multiple samples per switching cycle to calculate the average value. Therefore, the best option is to include a filter in the feedback to attenuate the current ripple.

Taking into account these considerations, the system can be modeled as in Fig.7. The current ripple will be considered as a perturbation $(w)$.

$$
\begin{gathered}
L=R \cdot G \cdot F \\
y=\frac{R \cdot G}{1+L} r+\frac{1}{1+L} w
\end{gathered}
$$

The design criterion for the two current loops has been the following: the cutoff frequency of the feedback filter is set at $100 \mathrm{~Hz}$ to have enough attenuation when the converter operates at low frequency $(10 \mathrm{kHz})$; the effect of the filter is taken into account to assure the stability of the system. A PI controller has been designed to be able to track the reference within the whole frequency range. The zero of the PI controller is set at the cutoff frequency of the filter. Consequently, the bandwidth of the current loops is $100 \mathrm{~Hz}$ and the phase margin is 65 degrees. Since the bandwidth is far from the minimum switching frequency, the effect of the delay of the digital control can be neglected.

The bode plot of both current control loops is shown in Fig. 8.

\section{B. Voltage Loops}

Once the current loops are designed and assuming that they behave as unit gain blocks by having a faster dynamics than the voltage loops, the voltage loops can be designed. From equations (12) and (13) we can obtain the plant of the voltage loops:

$$
\begin{gathered}
G_{1}(s)=\frac{V_{s}(s)}{i_{g}(s)}=\frac{V_{g}}{V_{s}^{r e f} C_{s} s} \\
G_{2}(s)=\frac{V_{o}(s)}{i_{L}(s)}=\frac{Z_{\text {load }}(s)}{1+Z_{\text {load }}(s) C_{o} s}
\end{gathered}
$$

As the conventional stationary frame regulators (usually PI) can only provide finite gain at nonzero frequencies, to obtain a zero steady-state error under regulating a sinusoidal signal a PR (Proportional Resonant) (18) controller has been designed to control the output voltage [8].

$$
R(s)=k_{p}+\frac{k_{i} s}{s^{2}+\omega^{2}}
$$




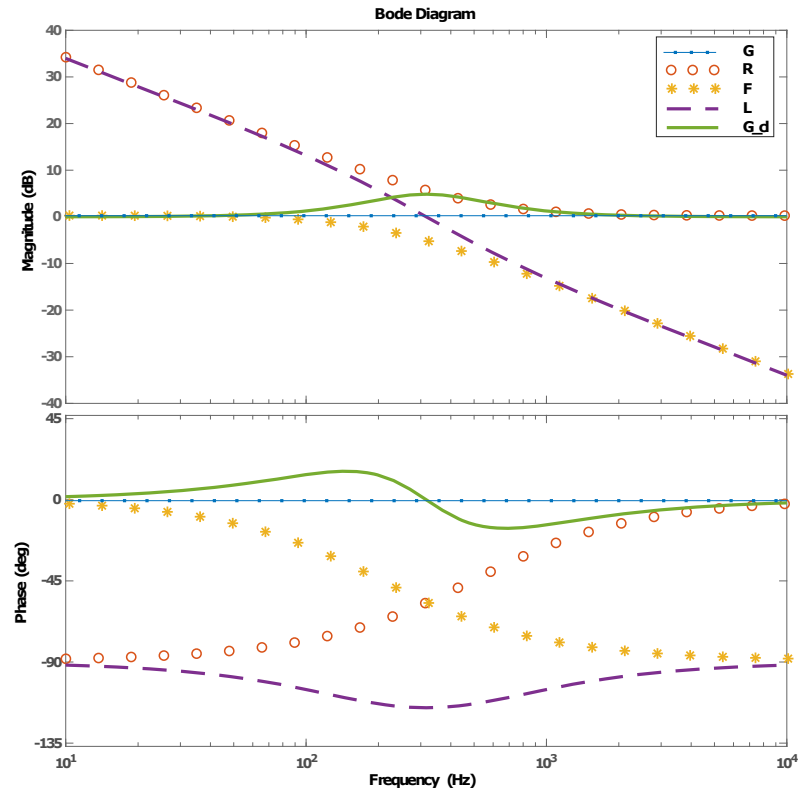

Figure 8. Bode plots of the different control loops: plant (blue), filter sensor (light blue), regulator (green), loop gain (red) and closed loop gain (magenta)

The control of this inverter will be implemented in an FPGA, to reduce the area and the processing time fixed-point arithmetic is used. As a result of the narrow band and infinite gain of PR controllers, special attention must be taken in the discretization [9]. In preference to the use of the shift-operator for the implementation of the PR controller, to reduce the finite word length effect [10]-[12] the $\delta$-operator is used. The delta operator $\delta$ can be defined in terms of the shift operator $z$ as:

$$
\delta^{-1}=\frac{\Delta z^{-1}}{1-z^{-1}}
$$

These two different implementations have been simulated with Matlab Simulink and the minimum size needed for all the coefficients have been calculated using the fixed-point tool. The $\delta$-operator allows using 16-bit word length resolution, instead of the 35-bit word length resolution needed for the shift transformation, which means less computation effort.

According to the control of the storage capacitor voltage, it should maintain the mean value neglecting the $120 \mathrm{~Hz}$ ripple. These specifications can be achieved with a low bandwidth. But if this capacitor is designed to handle only the unbalance between the input and the output power, after a load step it cannot deliver or store enough energy without changing dramatically its voltage during the transient. So, it is needed fast dynamics to adapt the input current after a load step to maintain the mean value. A good compromise between the input current ripple and the response under a load step would not possible without increasing the capacitance if the input current ripple is limited to the steady state ripple (20\%). The increase of the capacitance value affects the total volume of the converter. Considering the volume of the capacitor

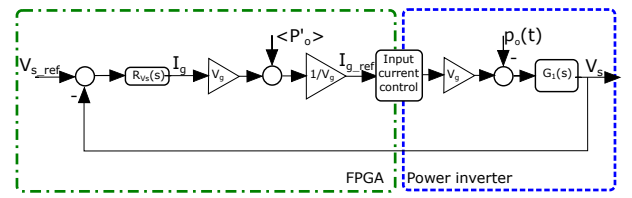

Figure 9. Storage capacitor voltage loop with active power estimation feedforward loop

proportional to the capacitance value, to reduce the volume maintaining a constant input current a high voltage discharge ratio in the capacitor is needed.

The proposed solution on this paper, Fig.9, consists on a slow dynamic feedback control loop, to maintain low input current ripple in steady state, and a feed-forward loop with the estimation of the active power delivered for the transients. In steady state, the active power will keep constant and the slow feedback loop will maintain a constant input current reference. Under a load step, the fast dynamic needed will be provided by the feed-forward loop.

The transfer functions of Fig. 9 are described in (20) and (21) and the loop gain can be expressed as in (22). To obtain a low input current ripple, only a low bandwidth control loop can be designed. The maximum bandwidth of the control loop can be calculated as a function of the maximum input ripple (23).

$$
\begin{gathered}
G(s)=\frac{1}{V_{c, \text { mean }} C_{s} s} \\
R(s)=k_{R}=i_{g, p p} / V_{s, p p} \\
L(s)=R \cdot G \cdot V_{g}=\frac{i_{g, p p}}{V_{s, p p}} \frac{V_{g}}{V_{c, \text { mean }}} \frac{1}{C_{s} s} \\
f_{B W, \text { max }}=\frac{i_{g, p p}}{I_{g}} f_{\text {line }}
\end{gathered}
$$

The converter under consideration is a $1 \mathrm{~kW}$ inverter with a $400 V_{D C}$ input voltage and $240 V_{r m s}$ at $60 \mathrm{~Hz}$ output voltage, the storage capacitor voltage has to be controlled around $0.75 V_{g}$ with a $100 \mathrm{~V}$ ripple. To comply with a $20 \%$ maximum input ripple the maximum bandwidth is $12 \mathrm{~Hz}$.

To obtain a fast estimation of the active power two different approaches have been analyzed.

The first idea is to use the dq synchronous reference frame transformation. Its main characteristic is that the fundamental components are mapped to only one dc signal. And another characteristic is that it can only be applied to three-phase systems [13]. One approach to extending the dq theory to single-phase converters is to use a quarter period time delayed version of the signal as the orthogonal vector.

Commonly, in single-phase system the dq transformation is used to implement synchronous frame controller [14], [15] because, such a controller can regulate the steady-state errors 
of the corresponding DC quantities to zero. But in this work, the $\mathrm{dq}$ transformation is used to calculate the active power.

$$
P_{o}=\operatorname{Re}\left\{V_{d q} I_{d q}\right\}=V_{d} I_{d}+V_{q} I_{q}
$$

This approach allows adapting the input current reference in a quarter period time. Producing a smaller variation in the storage capacitor voltage. Similar results can be obtained using the stationary reference frame $\alpha \beta$, reducing significantly the computational cost. As in the previous case, the orthogonal variable is obtained shifting $90^{\circ}$ the original signal, and transforming the output current and voltage into $\alpha \beta$ the output active power can be obtained as:

$$
P_{o}=\operatorname{Re}\left\{V_{\alpha \beta} I_{\alpha \beta}\right\}=V_{\alpha} I_{\alpha}+V_{\beta} I_{\beta}
$$

Therefore, the bandwidth of this control loop is $10 \mathrm{~Hz}$. A PI controller has been designed taking into account these considerations.

To compare both solutions with a conventional control without the feed-forward loop, a simplified Simulink model has been implemented. At 1s (Fig.10b), a load step occurs, changing the power factor from 1 to $\sqrt{3} / 2$ maintaining the apparent power. At $1.5 \mathrm{~s}$ (Fig.10c) the load maintains the power factor changing the apparent power from 850VA to 680VA. As shown in Fig.10a, similar results can be obtained using $\alpha \beta$ stationary reference frame (blue) or using dq rotating reference frame (green) in less than 3 line cycle the system reaches the steady-state, while a large deviation from the reference occurs with a conventional feedback loop (magenta), as expected.

Because the switching frequency is variable, this fact should be considered when discretizing the regulators. In order to facilitate the transformation of the continuous domain to the discrete one and thus to reduce the computational cost of the regulators, the Backward Euler method was chosen as conversion method, according to which the transformation of a PI regulator is defined as:

$$
\begin{aligned}
R(s) & =K_{p}+\frac{K_{i}}{s} \Rightarrow R(z)=\frac{K_{p}(z-1)+K_{i} t_{s}}{z-1} \\
i_{\text {ref }}(n) & =i_{\text {ref }}(n-1)+K_{i} t_{s}(n-1) V_{\text {error }}(n-1)+ \\
& +K_{p}\left[V_{\text {error }}(n)-V_{\text {error }}(n-1)\right]
\end{aligned}
$$

\section{Simulation And ExPerimental Results}

In this section, simulation and experimental results will be shown to prove the validity of the proposed control technique.

As mentioned before, the control was implemented in an FPGA in order to reduce the computation time required for the inversion of the plant and to implement the complex modulations of the inverter. It has been successfully validated with an experimental prototype.

Experimental results shown in Fig.11 and Fig.12 validate the concept, generating a $24 V_{r m s}, 60 \mathrm{~Hz}$, waveform, from a $40 V_{D C}$ input voltage, that is 10 times lower voltage but nominal current. The waveforms shown in Fig.11 are the sequence number in yellow and the output voltage in blue. Meanwhile, Fig. 12 shows a detail of the line cycle, when there is a change in the operation mode that can be noticed in the

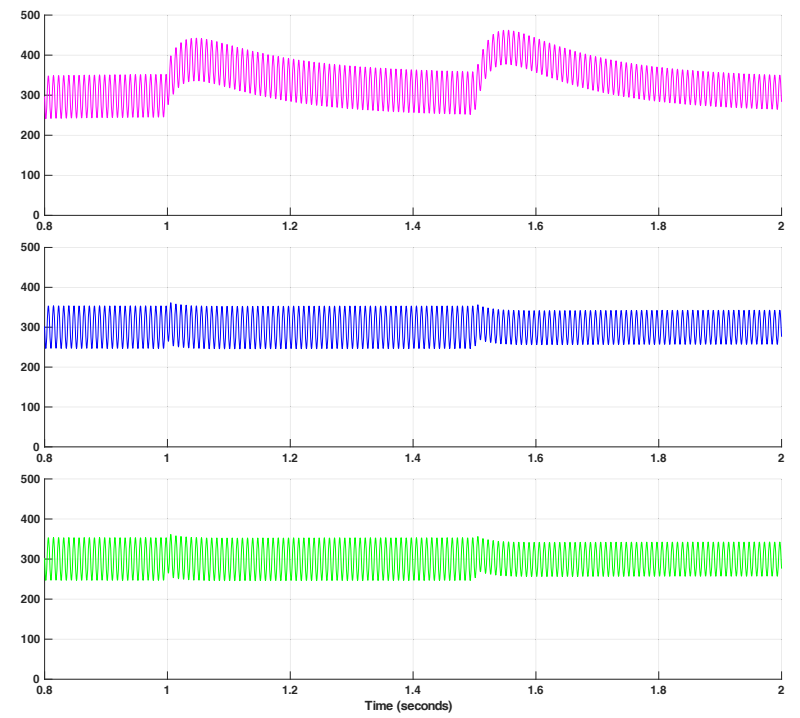

(a)

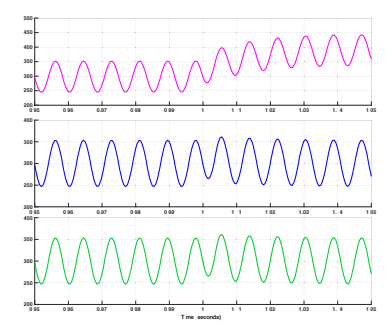

(b)

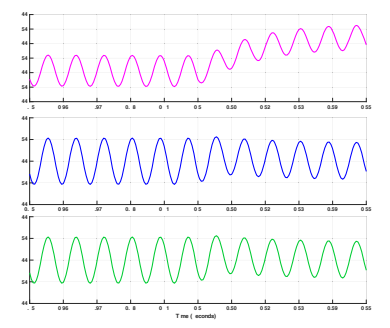

(c)
Figure 10. Simulation result of different storage capacitor voltage loops. From top to bottom: conventional low bandwidth control loop (magenta), control loop with active power estimation using dq (blue) and control loop with active power estimation using $\alpha \beta$ (green).

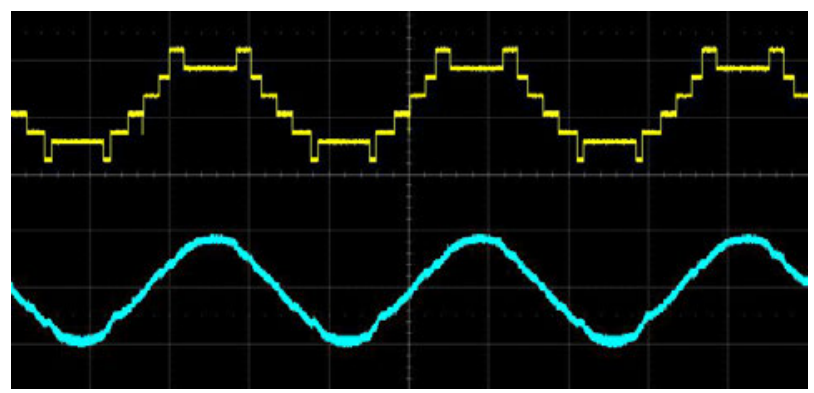

Figure 11. Experimental waveforms of the inverter: Output voltage (blue) Sequence number (yellow)

inductor current waveform; this change implies also a change in the switching frequency.

A new inverter for nominal voltage is being developed using high $d v / d t$ drivers for high speed $\mathrm{GaN}$ devices.

Fig.13 shows the simulated waveform of the inverter operating from $400 V_{D C}$ input voltage and generating an output voltage of $60 \mathrm{~Hz}$ and $240 V_{R M S}$. The top figure shows the output active power estimated. The second one represents the input current with a ripple lower than $15 \%$. The third one 


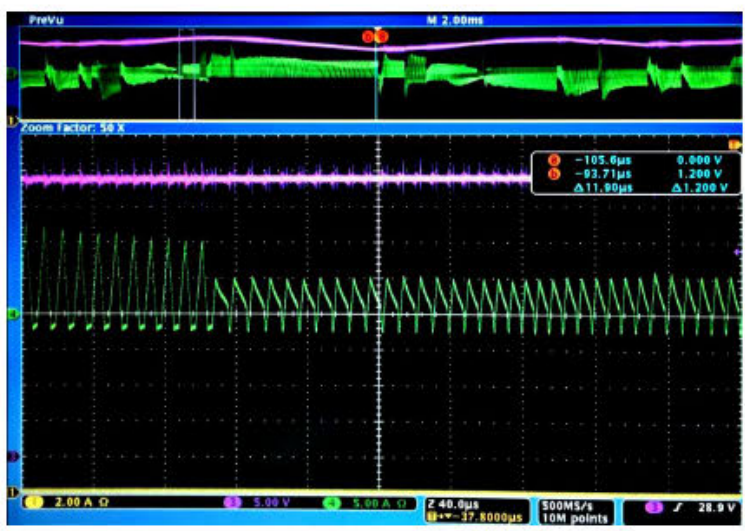

Figure 12. Experimental waveforms of the inverter: Output voltage (magenta) Inductor current (green)

shows the voltage of the three main ports, in blue it is shown the output voltage on top of the reference voltage (magenta), in red it is shown the storage capacitor voltage with the $120 \mathrm{~Hz}$ ripple due to the instantaneous unbalance between the input power, that is constant, and the output power that is sinusoidal, and the input voltage is represented in green. The bottom plot in Fig.13 shows a waveform that indicates the switching sequence that is applied. There are up to 19 different sequences that can be applied during the line cycle. All these sequences imply a completely different plant to be controlled during a short period of time. The proposed control, by means of the inversion of the plant, allows using two simple independent controllers for the output voltage and the storage capacitor. At $120 \mathrm{~ms}$ a load step occurs from $700 \mathrm{~W}$ to $1 \mathrm{~kW}$.The system recovers to the steady state in less than 3 line cycles. Figure 14 shows another simulation where can be seen the soft start to charge the storage capacitor without delivering the power to the load $(0-5 m s)$. After that the converter operate in closed loop regulating the input current and the output voltage. If during a transient period the storage capacitor voltage saturates at input voltage, the input current cannot be controller. At $120 \mathrm{~ms}$ a load step occurs from resistive load to inductive load $(\mathrm{PF}=0.7)$.

\section{CONCLUSION AND FUTURE WORK}

This work has shown that it is possible to control highly complex power converter topologies with multiple switching sequences by applying plant inversion technique. The technique has been applied to a highly compact single phase inverter in which different modulation strategies are applied along the line cycle in order to optimize the size and losses of the converter.

The proposed technique allows decoupling the control of the output voltage and the storage capacitor voltage and, what is more important, a very simple linear controller can be applied to all the sequences, what simplifies the control design.

The use of the feed-forward power estimation included in the storage capacitor voltage loop allows to maintain a low bandwidth control loop to reduce the input current ripple while

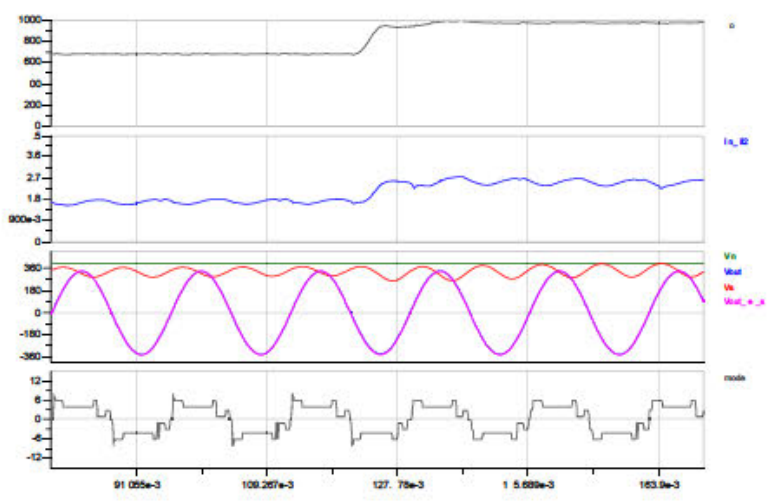

Figure 13. Simulated waveforms of the inverter. From top to bottom: Output power estimation, Input current, Output voltage (blue) input voltage (green) and storage capacitor (red), Sequence number applied

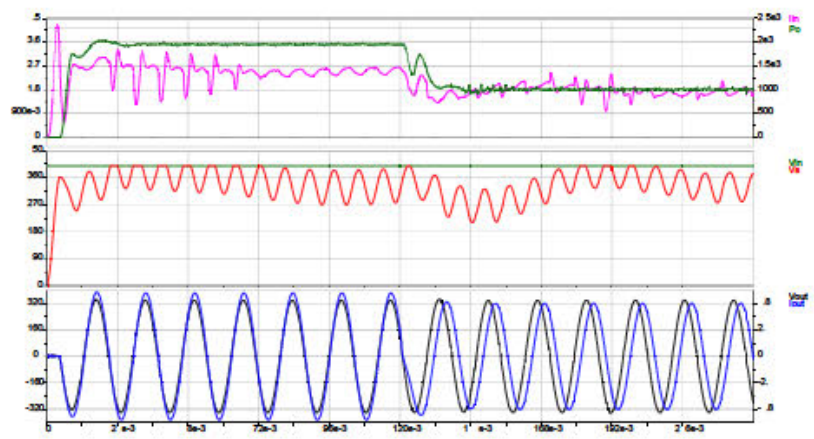

Figure 14. Simulated waveforms of the inverter. From top to bottom: Output power estimation, Input current, input voltage (green) and storage capacitor (red) and output voltage (black) and output voltage (current)

having a fast response to adapt the input current reference under load steps.

\section{ACKNOWLEDGMENT}

The authors would like to thank the Spanish Ministry of Education, Culture and Sport for its support under the FPU grant program (Reference FPU15/07081).

\section{REFERENCES}

[1] T. Meynard and H. Foch, "Multi-level conversion: high voltage choppers and voltage-source inverters," in Power Electronics Specialists Conference, 1992. PESC'92 Record., 23rd Annual IEEE, pp. 397-403, IEEE, 1992.

[2] J. A. Cobos, "Optimization of energy buffered converters," in Power Electronics (CIEP), 2016 13th International Conference on, IEEE, 2016.

[3] J. A. Cobos, H. Cristobal, D. Serrano, R. Ramos, J. A. Oliver, and P. Alou, "Differential power as a metric to optimize power converters and architectures," pp. 1-7.

[4] R. Ramos, I. Zubitur, D. Serrano, J. A. Oliver, P. Alou, and J. A. Cobos, "Mode selection strategy for multi-mode power converters to minimize its differential power," 2017.

[5] R. Middlebrook and S. Cuk, "A general unified approach to modelling switching-converter power stages," in Power Electronics Specialists Conference, 1976 IEEE, pp. 18-34, IEEE, 1976.

[6] P. Delarue, F. Gruson, and X. Guillaud, "Energetic macroscopic representation and inversion based control of a modular multilevel converter," in Power Electronics and Applications (EPE), 2013 15th European Conference on, pp. 1-10, IEEE, 2013. 
[7] J. M. Olm Miras, X. Ros Oton, and Y. Shtessel, "Stable inversionbased robust tracking control in dc-dc nonlinear switched converters," in 48th IEEE Conference on Decision \& Control \& 28th Chinese Control Conference, pp. 2789-2794, 2009.

[8] D. N. Zmood and D. G. Holmes, "Stationary frame current regulation of pwm inverters with zero steady-state error," IEEE Transactions on power electronics, vol. 18, no. 3, pp. 814-822, 2003.

[9] S. A. Khajehoddin, M. Karimi-Ghartemani, P. K. Jain, and A. Bakhshai, "A resonant controller with high structural robustness for fixed-point digital implementations," IEEE Transactions on Power Electronics, vol. 27, no. 7, pp. 3352-3362, 2012.

[10] M. J. Newman and D. G. Holmes, "Delta operator digital filters for high performance inverter applications," IEEE Transactions on Power Electronics, vol. 18, no. 1, pp. 447-454, 2003.

[11] R. Middleton and G. Goodwin, "Improved finite word length characteristics in digital control using delta operators," IEEE Transactions on Automatic Control, vol. 31, no. 11, pp. 1015-1021, 1986.
[12] D. Sera, T. Kerekes, M. Lungeanu, P. Nakhost, R. Teodorescu, G. K. Andersen, and M. Liserre, "Low-cost digital implementation of proportional-resonant current controllers for pv inverter applications using delta operator," in Industrial Electronics Society, 2005. IECON 2005. 31st Annual Conference of IEEE, pp. 6-pp, IEEE, 2005.

[13] M. Gonzalez, V. Cárdenas, and F. Pazos, "Dq transformation development for single-phase systems to compensate harmonic distortion and reactive power," in Power Electronics Congress, 2004. CIEP 2004. 9th IEEE International, pp. 177-182, IEEE, 2004.

[14] B. Crowhurst, E. El-Saadany, L. El Chaar, and L. Lamont, "Singlephase grid-tie inverter control using dq transform for active and reactive load power compensation," in Power and Energy (PECon), 2010 IEEE International Conference on, pp. 489-494, IEEE, 2010.

[15] M. J. Ryan and R. D. Lorenz, "A synchronous-frame controller for a single-phase sine wave inverter," in Applied Power Electronics Conference and Exposition, 1997. APEC'97 Conference Proceedings 1997., Twelfth Annual, vol. 2, pp. 813-819, IEEE, 1997. 